\title{
Intelligent Method for Dipstick Urinalysis Using Smartphone Camera
}

\author{
R.V. Hari Ginardi, Ahmad Saikhu, Riyanarto Sarno, Dwi Sunaryono, \\ Ali Sofyan Kholimi, and Ratna Nur Tiara Shanty \\ Department of Informatics, Institut Teknologi Sepuluh Nopember (ITS) Surabaya \\ hari@its.ac.id
}

\begin{abstract}
This paper introduces an intelligent method for helping people to maintain their healthy by doing a self urinalysis utilising a smartphone camera. A color sensing method using a smartphone camera is designed to determine the value of a reagent strip in a urinalysis dipstick. In the dipstick urinalysis, a color change in each reagent strip is examined. This color change is a result of the reaction of dipstick to the chemical contents of urine including $\mathrm{pH}$, Protein, Glucose, Ketones, Leucocyte, Nitrite, Bilirubine, and Urobilinogen. Performing disptick urinalysis can be done in almost any places even on the very remote area where medical laboratory cannot be found, and it is much easier and cheaper than medical lab visit.

The proposed intelligent method includes a framework for color acquisition using a smartphone camera which covers the color management system, color correction, color matching, and quantification. The usage of RGB and CIELAB color space is discussed in the color management part. An automated color constancy approach is introduced to provide a better color correction, and a step-wise linear interpolation is introduced to better estimate the urinalysis value.

To implement this proposed method, a closed acquisition box does not required. Disptick capturing can be done directly with a smartphone camera in almost any normal lighting condition.
\end{abstract}

Keywords: color sensing, dipstick urinalysis, color constancy.

\section{Introduction}

Many methods have been applied to reduce or control the blood sugar for diabetes mellitus (DM) patients. A periodic postprandial glucose test and fasting plasma glucose test are among the most commonly practiced. Those require the patient to visit a medical laboratory. The test itself requires a blood sample from patient which is taken using a needle. Besides these blood glucose tests, an examination of the concentration of glucose in patient urine is performed through a method called urinalysis.

Urinalysis has been largely used as a method to determine the presence of chemical composition in urine. Currently, urinalysis can be performed using urine dipstick reagents. It can be done by placing the dipstick into a collected sample of urine and 
visually comparing the reactive color of each reagent with the dipstick color chart based on their color similarities. This manual interpretation has its limitation, including differences in a perception of color, differences in the lighting condition [1] and limitation on categorical or semi-quantitative assessment only [2].

Dipstick reader is a device used by most laboratories to interpret the dipstick results automatically. This device can minimize errors caused by lighting condition and differences in color perception, significantly [3]. However, its price is expensive for individual treatment. Considering the possibility of most smartphones to capture color image using its digital camera, a smartphone could be an alternative device to interpret urine dipstick results.

Automatic dipstick interpretation using a smartphone camera requires a method for color acquisition and a method of color similarity measurement to determine the corresponding chemical content concentration. Incosistencies may occur in lighting condition, focus, and angle of view [4]. A color constancy technique is used to approximate the original color of the captured image [5],[6]. It enables consistent color acquisition.

In this research, a framework for intelligent dipstick color acquisition using a smartphone is proposed. We analyse the color constancy measurement for image acquisition using a smartphone camera in a daily lighting condition without a closed box, and choose the optimal color constancy method to provide the color correction. An approach for smoother color quantification is done by introducing a stepwise linear interpolation [7]. It gives a better approximation to determine the reagent score of urine substances with quantitative assessment such as glucose.

\section{Related Works}

\subsection{Color Space}

A color space is a mathematical model to specify and visualize colors. Several color spaces have been defined namely RGB, CIEXYZ, CIELAB, HSV, and CMYK.

Color perception by human visual is defined by three basic attributes. Those are hue, saturation, and brightness [8]. Hue defines the differences in specific tone of color; such as red, green blue, or yellow; brightness defines the level of lightness or darkness of a color, while saturation describes the purity of a color.

RGB is a standard color space used by electronic imaging devices such as monitor, camera, and scanner [9]. It consists of red, green, and blue components which are represented in a three-dimensional cube. Each component has a value ranged from 0 to 255 [10]. RGB does not represent how the human visual interprets colors [11].

CIELAB is a color space defined by CIE (Comission Internationale de l'Eclairage/International Commission on Illumination) in 1976. It is used in most of digital image processing, because it describes the representation of human visual interprets the color. 


\subsection{Color Constancy}

Digital color images which are taken by using a digital camera, is highly dependent on three things, they are illuminants, sensor sensitivity, and reflectance. It is represented by the following Equation 1.

$$
f c(x)=m(x) \int_{\omega} I(\lambda) \rho_{c}(\lambda) \mathrm{S}(\mathrm{x}, \lambda) \mathrm{d} \lambda,
$$

where $f c$ is the captured color, $I$ is illuminant, $\lambda$ is wavelength, $\rho_{c}$ is camera sensitivity, and $S$ is the ability for reflecting lightness from the object,

Assuming the sensitivity $\rho_{c}$ and the wavelength $\lambda$ in different types of camera are constant, those variables can be neglected, therefore the differences in color acquisition will be highly dependent on the varieties of illumination.

Color constancy is a process to restore an image into a certain illumination value even if it is captured in different illumination conditions [6]. Color constancy plays significant roles when color recognition or quantification is required in an image acquisition with varieties of illumination such as colorimetric analysis[12][13][14], and it is also applied in dipstick urine analysis.

In general, color constancy process is begun with an evaluation of lighting source color and its intensity to determine the required color correction. The result is an image in which its color is corrected as it was captured with a white source color.

White Patch Retinex is one of the color constancy method based on the amount of illumination. It is done by using white color as a ground truth. This color is used to adjust the level of illumination [6]. It ignores the differences on reflectance value of the object. Other methods are uniform, non-uniform, and learning method. Uniform method is capable for image enhancement purpose.

Based on its complexity, Gijsenij[6] divided color constancy algorithms into three methods, namely stastistical method, gamut method, and machine learning. Statistical method has a lower computing complexity, but it is less accurate in correcting image with high variance such as real photos.

Another method, Grey-World, is a uniform [11] and statistical method. This method failed to correct an image with one dominant color such as leaf color image [11]. Gerson [15] improves the Grey-World by applying a segmentation first. An illumination correction is performed differently for each segment.

Grey World and White Patch uses low level of RGB values of an image. Van de Weijer, et all [16] proposed the usage of high level RGB which is derived from low level RGB Worlds, which is then named Grey Edge algorithm. However, Grey Edge is not optimal for images with one dominant color.

\subsection{Color Distance Measurement}

Color similarity can be done by measuring the distance between points on a geometry space. Several techniques to calculate distances on a geometry space are Euclidean distance, angle vector distance, Canberra distance, and Minkowski. However, Canberra distance and Minkowski distance perform poorly in color similarity for an image retrieval research [17]. 
Vector angle distance and Euclidean distance are mostly used for measuring color distance. Hue and saturation differences can be quantified well using Vector angle distance. While Euclidean distance quantifies well illumination intensity. Since Euclidean distance has the lower computational complexity, we prefer this technique as a first step to determine the color similarity.

\section{$3 \quad$ Methodology}

\subsection{Proposed System}

Our proposed system is shown in Fig.1. A test dipstick is captured by using a smartphone camera. Due to the lighting condition and the characteristic of the camera sensor, the color in the produced digital image might be different with the original color of tested dipstick. Therefore, color constancy measurement is needed to approximate its original color and perform an automated color correction. For each image capture, the whole dipstick is captured, containing a series of ten or more reagent color. To separate each reagent color into an individual image file, a cropping process is required. Those reagent color can be organized in a RGB color space or CIELAB color space.

Quantification is done by matching the tested color with its corresponding color chart based on their color similarity and retrieve the related chemical value. For qualitative or semi-quantitative urine analysis, a color similarity approach is adequate, however a further analysis is required for an absolute value determination. Concerning the stepped value in the color chart, a stepwise linear interpolation method is proposed. This interpolation method can estimate a more precise measurement value.

The result from above color quantification is used by the health monitoring system application. A prototype of this application has been built in an Android platform.

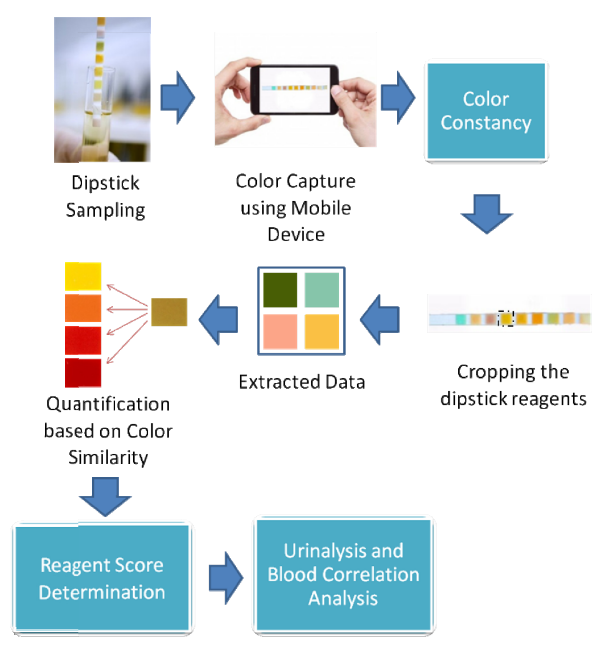

Fig. 1. Process Flow of the Proposed System 


\subsection{Color Constancy}

The proper color constancy method is obtained by evaluating the existing methods. Evaluation is done by measuring the distribution of colors on each reagent in a specific color space.

A statistical range of color distribution in a reagent image is chosen to evaluate how the result is spread or fold-up. Range is used rather than variance since it provides a wider result [18] and it is more sensitive to outlier.

White Patch Color Constancy method is done by getting the maximum value of color from each color component (R, G, and B) as described in Equation 2.

$$
K \max =\max (\max (R), \max (G), \max (B))
$$

After obtaining the maximum value, the ratio of each color component is calculated, as described in Equation 3.

$$
R c=\frac{\max (C)}{K \max },
$$

where $C$ is color of R,G,B color space.

After getting the ratio, the value range of each color component will be widened according to the ratio as described by Equation 4, in order to get the original color.

$$
I(X)=R_{c} \times F_{c}(X)
$$

As an improvement method from White-Patch, Grey-World [19] has a similar approach. Firstly it calculates statistical mean of each color element. An inversion is required as shown in Equation 5, where $C$ is a color from RGB color space.

$$
K(C)=\frac{1}{\operatorname{avg}(\operatorname{avg}(C))}
$$

The following Equation 6 shows the calculation of maximum value $\mathrm{K}$ from $\mathrm{K}(\mathrm{C})$.

$$
K \max =\max (K(R), K(G), K(B))
$$

The result of Kmax is used to find a ratio for scaling factor as given in Equation 7. This Equation is actually an inverse of Equation 3.

$$
R c=\frac{K \max }{\max (C)}
$$

Furthermore, as in White-Patch, this ratio value $R c$ is used in Equation 4. The pattern similarity between White-Patch and Grey-World shows that both methods are equivalent. They are originating from the same equation as shown in Equation 8, where $\mathrm{p}$ is equal to 1 for Grey-World and $p$ is equal to $\infty$ for White-Patch.

$$
\left.I(X)=\sum(F c(X))^{p}\right)^{\frac{1}{p}}
$$




\subsection{Quantitative Assessment}

By its nature, reagents in dipstick urinalysis provides result in a stepped values. Glucose reagent for example, has a specific color for value $0,2.8,5.5,14,28$ and $>=55$.

Comparing the color in the tested dipstick to the series of color in the dipstick color chart and finding its similarity will select one of the closest color as the quantification result. This method is used in most dipstick reader device.

To improve the accuracy of dipstick color interpretation, a curve fitting approach is considered. This curve is used to estimate the value if the tested color lies between two reference colors in the color chart as shown in the following Fig. 2.

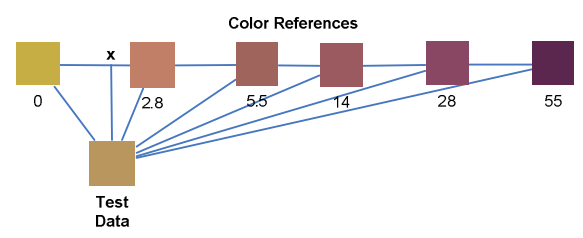

Fig. 2. Dipstick Color Interpretation

\subsection{Stepwise Linear Interpolation}

A stepwise linear interpolation is used to estimate the value between two given values. This interpolation is applied for each pair of neighboring color in the color chart. The distance between two colors in the color chart and the distance between a tested color and a color chart is evaluated in a cartesian coordinate system with three-elements of color space are used as the coordinate system. From the previous research[7], a CIELAB color space is chosen, therefore its color component $\mathrm{L}, \mathrm{a}, \mathrm{b}$ are mapped into a cartesian coordinate system. X, Y axis defines the level of a reagent, and the value of color component, respectively. Assessing the score of the tested reagent is described as follows:

1. Using euclidean distance, calculate the distance between two adjacent reference data, and the distance between a test data and each reference data using Equation 9, as shown in Fig. 3.

$$
d=\sqrt{(\Delta L)^{2}+(\Delta a)^{2}+(\Delta b)^{2}},
$$

where $\Delta L=L_{\text {test }}-L_{r e f}, \Delta a=a_{\text {test }}-a_{\text {ref, }} \Delta b=b_{\text {test }}-b_{\text {ref }}$

2. Find a node $n$ in reference data that has the shortest distance to test data. The value of $n$ represents the location of the selected data.

a. If $n-1$ is equal to 0 , then the node is located on the first level. Assign the node number $n$ as node "B" and the node number $n+1$ as node " $\mathrm{C}$ ". Go to step 3.

b. If $n+1$ is more than the numbers of reference data, then the node located is on the last level. Assign the node number $n$ as node " $\mathrm{C}$ " and the node number $n-1$ as node "B". Go to step 3 .

c. Otherwise, this node is between two nodes. Continue to step 4 . 
3. Check if the distance between test data to node "B" is longer than the distance between node "B" and node "C", OR the distance between test data and node "C" is longer than the distance between node " $\mathrm{B}$ " and node " $\mathrm{C}$ ", this test data is beyond the color chart range. Skip the next step and mark the test data as an error.

4. Calculate the distance of test data to node $n-1$ and the distance of test data to $n+1$. Find the shortest one and note the node.

5. If the resulted node is $n-1$, check whether the distance between test data and node $n-1$ is smaller than the distance between node $n-1$ and $n$. If it is true, denote $n-1$ as "B" and denote $n$ as " $\mathrm{C}$ ".

6. If the resulted node is $n+1$ check whether the distance between test data and node $n+1$ is smaller than the distance between node $n+1$ and $n$. If it is true, denote $n+1$ as " $C$ " and denote $n$ as "B".

7. If both of two conditions on step 5 and 6 above are not fulfilled, this test data is beyond the color chart range. Skip the next step and mark the test data as an error.

8. As shown in Fig. 4, the relationship between test data, B, and C is evaluated using trigonometry model to project the current test node position into its relative position between node B and C. Equation 6 is used for this evaluation model.

9. The tested reagent score can be assessed using Equation 11.

$$
\begin{aligned}
& C x=\frac{A B^{2}-B C^{2}-A C^{2}}{-2 B C} \\
& \left(\frac{B x}{B c} \times(\text { ScoreofC }- \text { Scoreof } B)\right)+\text { Scoreof } B
\end{aligned}
$$

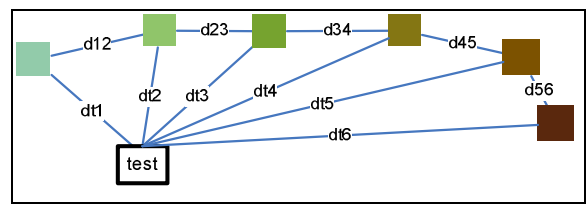

Fig. 3. Test Data and Reference Data Distances Calculation

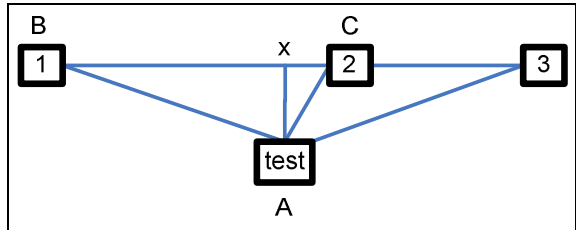

Fig. 4. The Relationship between Test Data, B, and C 


\section{$4 \quad$ Results and Analysis}

\subsection{Color Constancy}

As shown in Table 2, White Patch technique able to reduce the range of inconsistency up to $63 \%$ of red component, $88 \%$ of green component, and $59 \%$ of blue component.

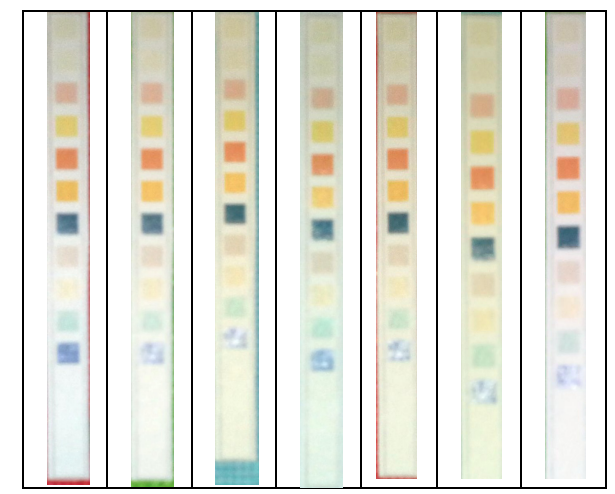

Fig. 5. Color Constancy using White Patch

Table 1. Color Constancy Result Using White Patch

\begin{tabular}{|l|r|r|r|}
\hline & \multicolumn{1}{|c|}{ R } & \multicolumn{1}{|c|}{ G } & \multicolumn{1}{|c|}{ B } \\
\hline Original & $\mathbf{5 1}$ & $\mathbf{5 1}$ & $\mathbf{4 9}$ \\
\hline Grey World & 50 & 52 & 53 \\
\hline White Patch* & $19^{*}$ & $6 *$ & $20^{*}$ \\
\hline Shades of Grey & 51 & 52 & 50 \\
\hline Grey Edge & 98 & 99 & 39 \\
\hline Gamut Mapping X-Derivation & 93 & 105 & 46 \\
\hline Gamut Mapping Y-Derivation & 86 & 113 & 45 \\
\hline Gamut Mapping 1st Order & 104 & 111 & 40 \\
\hline
\end{tabular}

\subsection{Color Quantification Result for Glucose Reagent}

A glucose estimation is chosen for color interpretation using our approach. A series of test data and reference data considered as the real color from reagent is prepared in Table 3. The reagent score assessment in ten samples is shown in Table 4. We provide quantitative assessment resulted from our approach, assessment by dipstick reader, and interpretation by human visual. The interpretation results produced by dipstick reader and human visual are in categorical values only, therefore the results of quantitative assessment cannot be compared exactly. Furthermore, we provide qualitative assessment to be evaluated. 
Outlier data might occur from wrongly captured reagent or out-of-range colors as shown in Table 5, and it should be detected. We evaluate the assessment using our approach by comparing with the assessment using Euclidean distance and human visual. The system is able to determine the score of glucose for the first type of real data and recognise the rest as unwanted or outlier colors.

\subsection{Reagent Score Assessment Using Proposed Approach}

In this section, a test data as shown in Table 3 is used as an example to be measured by the formula resulted from our algorithm. The score is assessed and described by Equation 12 and 13. The trigonometry model is illustrated in Fig. 6. Proportional position determination of the tested data is shown in Fig. 7.

$$
C x=\frac{21.35^{2}-42.94^{2}-22.83^{2}}{-2 \times 42.94}=22.23
$$

Score of Glucose $=$

$$
\left(\frac{20.704}{42.94} \times(2.8-0)\right)+0=1.3502
$$

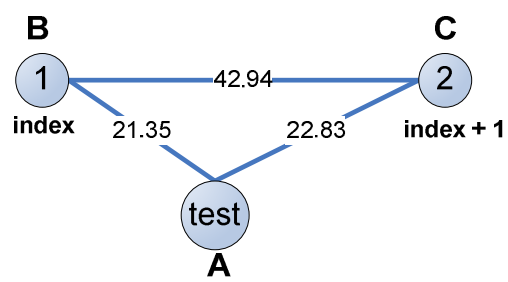

Fig. 6. The Relationship between Test Data, Reference Data 1, and 2

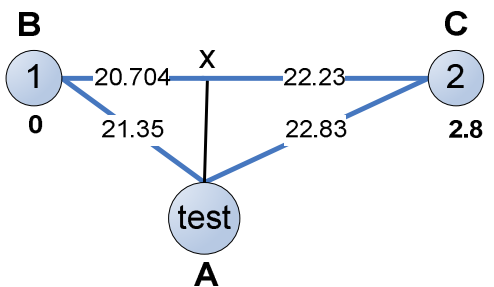

Fig. 7. Proportional position determination of the tested data 
Table 2. Interpretation Result of Glucose Reagent

\begin{tabular}{|c|c|}
\hline $\begin{array}{c}\begin{array}{c}\text { Level } \\
(\mathrm{mmol} / \mathrm{L})\end{array} \\
\end{array}$ & $\begin{array}{c}\text { Reference } \\
\text { Data }\end{array}$ \\
\hline $\begin{array}{c}- \\
(\mathbf{0})\end{array}$ & \\
\hline $\begin{array}{c} \pm \\
(2.8)\end{array}$ & \\
\hline $\begin{array}{c}+ \\
(5.5)\end{array}$ & \\
\hline $\begin{array}{l}++ \\
(14)\end{array}$ & \\
\hline $\begin{array}{l}+++ \\
(28)\end{array}$ & \\
\hline $\begin{array}{l}++++ \\
(\geq 55)\end{array}$ & \\
\hline Test Data & \\
\hline Visual & $-(0)$ \\
\hline $\begin{array}{c}\text { Dipstick } \\
\text { Reader }\end{array}$ & $-(0)$ \\
\hline Quantitative & 1.35 \\
\hline
\end{tabular}

Table 3. Score Assessment Comparison in Several Samples

\begin{tabular}{|c|c|c|c|c|}
\hline Sample & Quantitative & Qualitative & Dipstick Reader & Visual \\
\hline 1 & 1.35 & $-(0)$ & $-(0)$ & $-(0)$ \\
\hline 2 & 1.16 & $-(0)$ & $-(0)$ & $-(0)$ \\
\hline 3 & 1.24 & $-(0)$ & $-(0)$ & $-(0)$ \\
\hline 4 & 1.20 & $-(0)$ & $-(0)$ & $-(0)$ \\
\hline 5 & 1.32 & $-(0)$ & $-(0)$ & $-(0)$ \\
\hline 6 & 26.73 & $+++(28)$ & $+++(28)$ & $+++(28)$ \\
\hline 7 & 42.12 & $++++(\geq 55)$ & $++++(\geq 55)$ & $++++(\geq 55)$ \\
\hline 8 & 36.39 & $++++(\geq 55)$ & $++++(\geq 55)$ & $++++(\geq 55)$ \\
\hline 9 & 2.23 & $\pm(2.8)$ & $\pm(2.8)$ & $\pm(2.8)$ \\
\hline 10 & 1.52 & $-(0)$ & $-(0)$ & $-(0)$ \\
\hline
\end{tabular}


Table 4. Interpretation Comparison Result of Outlier Data

\begin{tabular}{|c|c|}
\hline $\begin{array}{c}\text { Level } \\
(\text { mmol/L) }\end{array}$ & $\begin{array}{c}\text { Reference } \\
\text { Data }\end{array}$ \\
\hline $\mathbf{1}$ & \\
\hline $\mathbf{3}$ & \\
\hline $\mathbf{4}$ & \\
\hline $\mathbf{5}$ & \\
\hline $\mathbf{6}$ & \\
\hline $\begin{array}{c}\text { Test Data } \\
\text { Stepwise } \\
\text { Linear } \\
\text { Interpolation }\end{array}$ & \\
\hline Visual & Outlier \\
\hline $\begin{array}{c}\text { Euclidean } \\
\text { Distance }\end{array}$ & Outlier \\
\hline \multicolumn{2}{|c|}{1} \\
\hline
\end{tabular}

\section{Conclusion}

Using the proposed intelligent method for dipstick urinaysis, a self monitoring of blood sugar of diabetes mellitus patient can be done without examining the patient blood. This approach helps people in remote areas which have limited access to the hospital or medical labratory to maintain their health. Evenmore, this approach does not require any physical intervention compare to blood test, makes it painless alternative for blood sugar control. With the highly availability and cheaper dipstick reagent strips, the operational cost of this method is considered cheaper than bood test.

The accuracy of the color quantification using this digital approach is very high makes the automatic color acquisition using smartphone is possible, even in the various lighting conditions.

The further improvement is a challenge to improve the color constancy performance with an automatic color calibration or using a relative calibration technique.

Acknowledgment. This research is supported by Japan Indonesia Corporation Agency (JICA)-Predicts phase-2, group B2-6 and a research grant from the Ministry of Education and Culture, The Republic of Indonesia 


\section{References}

1. Peele, J.D.: Evaluation of Ames Clini-Tek. Clinical Chemistry 23(12), 2238-2241 (1997)

2. Penders, J., Fiers, T., Delanghe, J.R.: Quantitative Evaluation of Urinalysis Test Strips. Clinical Chemistry 48(12), 2236-2241 (2002)

3. Tighe, P.: Laboratory-based quality assurance programme for near-patient urine dipstick testing. 1990-1997: development, management and results. Br. J. Biomed. Sci. 56, 6-15 (1999)

4. Capurso, J.: Mobile Document Capture: Scanner vs. Phone Camera. Xerox White Paper (2012)

5. Bianco, S., Ciocca, G., Cusano, C., Schettini, R.: Automatic color constancy algorithm selection and combination. Journal of Pattern Recognition 43 (2010)

6. Gijsenij, A., Gevers, T., Van De Weijer, J.: Computational color constancy: Survey and experiments. IEEE Transactions on Image Processing 20(9) (2011)

7. Shanty, R.N.T., Hari Ginardi, R.V., Sarno, R.: Interpretation of Urine Dipstick Results Based On Color Similarity Using Linear Interpolation Curve Fitting. In: IEEE International Conference on Computational Intelligence and Cybernetics, pp. 36-39 (2013)

8. Burns, B., Shepp, B.E.: Dimensional interactions and the structure of psychological space: The representation of hue, saturation, and brightness. Perception \& Psychophysics 43(5), 494-507 (1988)

9. Süsstrunk, S., Buckley, R., Swen, S.: Standard RGB Color Spaces. In: The Seventh Color Imaging Conference: Color Science, Systems, and Applications, pp. 127-134 (1999)

10. Pascale, D.: A Review of RGB Color Spaces. The BabelColor Company, 5700 Hector Desloges, Montreal (Quebec), Canada H1T $3 Z 6$ (2003)

11. CIE (Commission Internationale de l'Eclairage) (1978); Recommendations on Uniform Color Spaces. Color Difference Equations, Psychometric Color Terms (suppl. 2). CIE Publication, Paris Cedex 16, Paris, France, 68 (1791)

12. Iqbal, Z., Bjorklund, R.B.: Colorimetric analysis of water and sand samples performed on a mobile phone. Talanta 84(4), 1118-1123 (2011)

13. García, A., et al.: Mobile phone platform as portable chemical analyzer. Sensors and Actuators B: Chemical 156(1), 350-359 (2011)

14. Bianco, S., Schettini, R.: Color constancy using faces. In: 2012 IEEE Conference on Computer Vision and Pattern Recognition (CVPR). IEEE (2012)

15. Gershon, R., Jepson, A.D., Tsotsos, J.K.: From [R, G, B] to Surface Reflectance: Computing Color Constant Descriptors in Images. IJCAI (1987)

16. Van De Weijer, J., Gevers, T., Gijsenij, A.: Edge-based color constancy. IEEE Transactions on Image Processing 16(9), 2207-2214 (2007)

17. Androutsos, D., Plataniotis, K.N., Venetsanopoulos, A.N.: Distance measures for color image retrieval. In: IEEE Intrnational Conference on Image Processing, Chicago (1998)

18. Ebner, M.: Color constancy, vol. 6 (2007), http: / /Wiley.com

19. Buchsbaum, G.: A spatial processor model for object colour perception. Journal of the Franklin Institute 310(1), 1-26 (1980) 